\title{
Hyperbolicity of scalar-tensor theories of gravity
}

\author{
Marcelo Salgado, ${ }^{1, *}$ David Martínez-del Río, ${ }^{1, \dagger}$ Miguel Alcubierre, ${ }^{1, \ddagger}$ and Darío Núñez ${ }^{1,2, \S}$ \\ ${ }^{1}$ Instituto de Ciencias Nucleares, Universidad Nacional Autónoma de México, Apartado Postal 70-543, México D.F. 04510, México \\ ${ }^{2}$ Max-Planck Institut für Gravitationsphysik, Albert Einstein Institut, 14476 Golm, Germany
}

(Received 14 January 2008; published 9 May 2008)

\begin{abstract}
Two first order strongly hyperbolic formulations of scalar-tensor theories of gravity allowing nonminimal couplings (Jordan frame) are presented along the lines of the $3+1$ decomposition of spacetime. One is based on the Bona-Massó formulation, while the other one employs a conformal decomposition similar to that of Baumgarte-Shapiro-Shibata-Nakamura. A modified Bona-Massó slicing condition adapted to the scalar-tensor theory is proposed for the analysis. This study confirms that the scalar-tensor theory has a well-posed Cauchy problem even when formulated in the Jordan frame.
\end{abstract}

DOI: 10.1103/PhysRevD.77.104010

PACS numbers: 04.50.-h, 04.20.Ex, 04.25.D-, 95.30.Sf

\section{INTRODUCTION}

Scalar-tensor theories of gravity (STT) are alternative theories of gravitation where a scalar field is coupled nonminimally to the curvature associated with the physical metric (this is the so-called Jordan frame representation). The term "physical metric" refers to a situation where test particles follow the geodesics of that metric. The variation of the action of the STT with respect to the physical metric gives rise to field equations which contain an effective energy-momentum tensor (EMT) involving second order derivatives in time and space of the scalar field. Such EMT has the property that "ordinary matter," i.e., matter associated with fields other that the scalar field, obeys the (weak) equivalence principle which mathematically translates into a conserved EMT for ordinary matter alone.

Since a priori, it was not clear how such second order derivatives could be eliminated (in terms of lower order derivatives), or managed so as to obtain a quasilinear system of hyperbolic equations for which the Cauchy problem was well-posed (in the Hadamard sense), many people decided to abandon this approach in favor of the socalled Einstein frame representation where the nonminimal coupling is absorbed into the curvature by means of a conformal transformation of the metric. The new conformal metric is unphysical in the sense that (non-null) test particles do not follow the geodesics of that metric. However, the mathematical advantage is that the field equations for the nonphysical metric resemble the standard Einstein field equations with an unphysical effective EMT which involves at most first order derivatives of a suitable transformed scalar field (this EMT is unphysical because the "ordinary matter" part is not separately conserved). In the Einstein frame one can show that by using standard gauges (e.g., harmonic gauge) the field equations acquire

\footnotetext{
*marcelo@nucleares.unam.mx

†david.martinez@nucleares.unam.mx

*malcubi@nucleares.unam.mx

§nunez@nucleares.unam.mx
}

the form required in the application of theorems that establish the well-posedness of the Cauchy problem.

In view of the apparent mathematical advantages and disadvantages of the Jordan and Einstein frames, several widespread misconceptions became common in the literature. One of these concerned the statement that the Cauchy problem is only well-posed in the Einstein frame [1]. In Ref. [2], however, one of us showed that this is not the case by following two different approaches. One was in the spirit of a second order analysis consisting on reducing the set of field equations, both for the metric components and the scalar field, to a manifestly quasilinear diagonal second order hyperbolic form (the "reduced" field equations). This was achieved by manipulating the field equations in a way that allowed one to express the d'Alambertian on the scalar field in terms of at most first order derivatives, and also by implementing a modified harmonic gauge which was adapted to the STT. Such a modified gauge allowed one to eliminate the remaining second order derivatives of the scalar field which had previously prevented the applicability of Leray's theorem (see, e.g., Ref. [3] for the theorem).

The second approach followed in [2], and which was directly related with the initial value problem, consisted in recasting the field equations of the STT in a $3+1$ or Arnowitt-Deser-Misner (ADM) [4] form written à la York [5] (hereafter referred to as the ADMY equations). To do so it was necessary to define suitable variables, followed by a manipulation of the equations in order to obtain well-defined constraints (independent of the choice of the lapse and shift), as well as first order in time evolution equations for the extrinsic curvature and the scalar-field variables. In Ref. [2], the main goal was to focus in the $3+1$ approach rather than the second order one because of the subsequent numerical applications we had in mind. In fact, almost all the modern codes used in numerical relativity are based on first order in time formulations, and therefore we wanted to adapt them for the analysis of phenomena in STT. 
Now, when taking the limit of pure general relativity (GR) (i.e., no no-minimal coupling), the $3+1$ equations presented in [2] reduce to the standard ADMY equations with a minimally coupled scalar field plus ordinary matter sources. It is well-known that the ADMY equations are not strongly hyperbolic (see [6] for a detailed discussion), and therefore the corresponding equations for STT described in [2] were expected not to be strongly hyperbolic either. As already mentioned in [2], such equations were only the first step towards a first order strongly hyperbolic system for which the well-posedness of the Cauchy problem could be established.

The aim of this paper is then to fill that gap, and to obtain a first order strongly hyperbolic system of partial differential equations based on the $3+1$ equations of [2]. Actually, we will show here two such systems: one that is related to the Bona-Massó-Seidel-Stela (BMSS) formulation [7] (which in turn is based on the Bona-Massó (BM) formulation [8]) and a second one based on the BaumgarteShapiro-Shibata-Nakamura (BSSN) conformal decomposition $[9,10]$. As was done in [2], a modified BM slicing condition will be used for the STT, while the shift vector will be taken as an a priori given function of the coordinates.

A last comment about the Jordan vs Einstein frame is in order. In [2] it was a matter of principle to show that the Cauchy problem could be well formulated in the Jordan frame. It then became clear that there was no fundamental reason to continue using the Einstein frame. After all, the Jordan frame is the one associated with the (physical) quantities that are to be confronted with the observations. Moreover, the constraint and evolution equations are not more involved than those of usual GR, so from a numerical point of view the use of the Jordan frame does not add much complexity to the analysis. On the other hand, the direct use of the Jordan frame allows a better physical interpretation of the results and avoids the potential problems that might arise in the Einstein frame in cases when the conformal transformations back to the physical metric are not well-defined. Finally, it has been argued that some of the energy densities associated with the Jordan frame effective EMT (JFEMT) are not positive definite and may therefore be unphysical, and also the corresponding ADM mass could then become negative. While it is certainly true that the JFEMT does not satisfy the energy conditions in general, it is very likely that any physical configuration consistent with observations will not carry (total) negative energy. This is because the deviations of any alternative theory from GR are presumably very small in order to reproduce many of the current observations (cf. Ref. [11]). Actually, in any viable STT cosmology it turns out that the total energy density of the Universe is always positive, even when taking into account the negative contributions due to the nonminimal coupling (NMC) [12-15]. Again, this is because the scalar-field contribu- tions (positive or negative) to the total energy density should be in agreement with several observations related to the past and present history of the Universe. For instance, the expansion rate of the Universe modified by the NMC contributions has to be consistent with the one required to produce the correct abundance of primordial nucleosynthesis [12]. In addition, the density perturbations in STT should also match the cosmic microwave background data. In other words, a STT cosmology which produces a total effective negative energy density of the Universe at any given epoch will surely not be consistent with observations. By the same token, in a consistent STT cosmology the negative contributions (if any) are to be naturally suppressed by the positive ones, providing a consistent universe.

In the case of astrophysical applications (e.g., neutron star models) within a class of STT with a positive definite NMC function (which implies a positive definite effective gravitational "constant"), the ADM mass turns to be always positive despite the negative contributions to the energy density due to the NMC [16]. Moreover, the value of the NMC cannot be very high as otherwise the corresponding STT would put in jeopardy the agreement with the binary pulsar observations [17].

Of course, the nice thing about matter fields satisfying the energy conditions is the applicability of several theorems (e.g., positive mass theorems and singularity theorems; see [3] and references therein). However, the fact that the JFEMT does not satisfy the energy conditions in general simply indicates that the theorems cannot say anything about the positivity of mass or the formation of singularities in this case. This is not a problem of physical but of mathematical character. But again, the nonpositive definite terms arising from the NMC will be surely bounded if the STT in hand is to be consistent with the current observations and experiments, and therefore the nice features of an EMT respecting the energy conditions will also very likely appear in any observationally consistent STT. Of course, all these arguments are only sustained by numerical experiments when constructing viable phenomenological models (in cosmology and compact objects), and therefore do not constitute a theorem. It would then be quite interesting to explore the possibility of proving positive energy and singularity theorems in the Jordan frame if one can bound the nonpositive definite contribution associated with the JFEMT.

This paper is organized as follows. In Sec. II we introduce the STT and the $3+1$ equations described in [2]. The notation of several variables will be slightly modified relative to Ref. [2] in order to match with the one usually employed in numerical relativity. In Sec. III, a fully first order strongly hyperbolic system of the field equations of STT is obtained along the lines of the BMSS and BSSN formulations of GR. We conclude with a discussion of future numerical applications of the hyperbolic systems 
presented here. Finally, in Appendix A we include the complete set of equations for both formulations, and in Appendix B we introduce a simple example to complement the ideas of Sec. III.

\section{SCALAR-TENSOR THEORIES OF GRAVITY}

The general action for STT with a single scalar field is given by

$$
\begin{aligned}
S\left[g_{a b}, \boldsymbol{\phi}, \boldsymbol{\psi}\right]= & \int\left\{\frac{F(\phi)}{16 \pi G_{0}} R-\frac{1}{2}(\nabla \phi)^{2}-V(\phi)\right\} \sqrt{-g} d^{4} x \\
& +S_{\text {matt }}\left[g_{a b}, \boldsymbol{\psi}\right],
\end{aligned}
$$

with $\phi$ the nonminimally coupled scalar field, and where $\psi$ represents collectively the matter fields, i.e., fields other than $\phi$, and $G_{0}$ is the usual gravitational constant (we use units such that $c=1$ ).

The representation of the STT given by Eq. (2.1) is called the Jordan frame representation. The field equations obtained from the action (2.1) are given by [18]

$$
\begin{gathered}
G_{a b}=8 \pi G_{0} T_{a b}, \\
\square \phi+\frac{1}{2} f^{\prime} R=V^{\prime},
\end{gathered}
$$

where ' indicates $\partial_{\phi}, \square:=g^{a b} \nabla_{a} \nabla_{b}$ is the covariant d'Alambertian operator, $G_{a b}=R_{a b}-\frac{1}{2} g_{a b} R$, and

$$
\begin{gathered}
T_{a b}:=\frac{G_{\mathrm{eff}}}{G_{0}}\left(T_{a b}^{f}+T_{a b}^{\phi}+T_{a b}^{\mathrm{matt}}\right), \\
T_{a b}^{f}:=\nabla_{a}\left(f^{\prime} \nabla_{b} \phi\right)-g_{a b} \nabla_{c}\left(f^{\prime} \nabla^{c} \phi\right), \\
T_{a b}^{\phi}:=\left(\nabla_{a} \phi\right)\left(\nabla_{b} \phi\right)-g_{a b}\left[\frac{1}{2}(\nabla \phi)^{2}+V(\phi)\right], \\
G_{\mathrm{eff}}:=\frac{1}{8 \pi f}, \quad f:=\frac{F}{8 \pi G_{0}} .
\end{gathered}
$$

Using Eq. (2.2), the Ricci scalar can be expressed in terms of the energy-momentum tensor Eq. (2.4). Equation (2.3) then takes the following form,

$$
\phi=\frac{f V^{\prime}-2 f^{\prime} V-\frac{1}{2} f^{\prime}\left(1+3 f^{\prime \prime}\right)(\nabla \phi)^{2}+\frac{1}{2} f^{\prime} T_{\text {matt }}}{f\left(1+\frac{3 f^{\prime 2}}{2 f}\right)},
$$

where $T_{\text {matt }}$ stands for the trace of $T_{\text {matt }}^{a b}$ (the subscript "matt" refers to matter fields other that $\phi$ ).

Now, the Bianchi identities imply that

$$
\nabla_{c} T^{c a}=0 .
$$

However, the use of the field equations leads, as mentioned before, to the conservation of the EMT of the matter alone

$$
\nabla_{c} T_{\text {matt }}^{c a}=0,
$$

which implies the fulfillment of the (weak) equivalence principle.

In what follows, we shall use the $3+1$ formalism of spacetime $[5,19]$ in order to recast the field equations as a Cauchy initial value problem. The following quantities turn out to be useful in the $3+1$ decomposition of the field equations of STT [2]

$$
\begin{aligned}
Q_{a} & :=D_{a} \phi, \\
\Pi: & =\mathcal{L}_{n} \phi,
\end{aligned}
$$

where $\mathcal{L}_{n}$ stands for the Lie derivative along the normal $n^{a}$ to the spacelike hypersurfaces $\Sigma_{t}$, and the operator $D_{a}$ is the covariant derivative compatible with the 3-metric $\gamma_{a b}$ induced on $\Sigma_{t}$. In components we have $n^{a}=$ $\left(1 / \alpha,-\beta^{i} / \alpha\right)$, where $\alpha$ and $\beta^{i}$ are the lapse function and shift vector, respectively, associated with the spacetime coordinates $\left(t, x^{i}\right)$ [20].

From definitions (2.11) and (2.12) it is possible to write an evolution equation for $Q_{a}$

$$
\mathcal{L}_{n} Q_{a}=\frac{1}{\alpha} D_{a}(\alpha \Pi) .
$$

On the other hand, the definition of the extrinsic curvature is given by

$$
K_{a b}:=-\frac{1}{2} \mathcal{L}_{n} \gamma_{a b}
$$

In this way, the $3+1$ decomposition of Eqs. (2.2) leads to a set of constraint equations plus the evolution equations for the extrinsic curvature (see Ref. [2] for details). The Hamiltonian and momentum constraints are respectively,

$$
\begin{aligned}
{ }^{3} R+ & K^{2}-K_{i j} K^{i j}-\frac{2}{f}\left[f^{\prime}\left(D_{l} Q^{l}+K \Pi\right)\right. \\
& \left.+\frac{\Pi^{2}}{2}+\frac{Q^{2}}{2}\left(1+2 f^{\prime \prime}\right)\right]=\frac{2}{f}\left[E_{\text {matt }}+V(\phi)\right],
\end{aligned}
$$

$$
\begin{aligned}
D_{l} K_{i}^{l} & -D_{i} K+\frac{1}{f}\left[f^{\prime}\left(K_{i}^{l} Q_{l}+D_{i} \Pi\right)+\Pi Q_{i}\left(1+f^{\prime \prime}\right)\right] \\
& =\frac{1}{f} J_{i}^{\text {matt }},
\end{aligned}
$$

where we have denoted $Q^{2}=Q_{l} Q^{l}, E_{\text {matt }}:=n^{a} n^{b} T_{a b}^{\text {matt }}$ and $J_{\text {matt }}^{a}:=-n_{d} \gamma^{a}{ }_{c} T_{\text {matt }}^{c d}$, and where $\gamma^{a}{ }_{c}=\delta^{a}{ }_{c}+n^{a} n_{c}$ is the projector onto $\Sigma_{t}$. The evolution equation for the extrinsic curvature takes the form 


$$
\begin{aligned}
& \partial_{t} K_{j}^{i}-\beta^{l} \partial_{l} K^{i}{ }_{j}-K^{i}{ }_{l} \partial_{j} \beta^{l}+K_{j}^{l} \partial_{l} \beta^{i}+D^{i} D_{j} \alpha-{ }^{3} R_{j}^{i} \alpha-\alpha K K_{j}^{i}+\frac{\alpha}{f}\left[Q^{i} Q_{j}\left(1+f^{\prime \prime}\right)+f^{\prime}\left(D^{i} Q_{j}+\Pi K_{j}^{i}\right)\right] \\
& -\frac{\delta_{j}^{i} \alpha}{2 f\left(1+\frac{3 f^{\prime 2}}{2 f}\right)}\left(Q^{2}-\Pi^{2}\right)\left(\frac{f^{\prime 2}}{2 f}-f^{\prime \prime}\right) \\
& =-\frac{\alpha}{2 f\left(1+\frac{3 f^{\prime 2}}{2 f}\right)}\left\{2 S_{\text {matt } j}^{i}\left(1+\frac{3 f^{\prime 2}}{2 f}\right)+\delta_{j}^{i}\left[f^{\prime} V^{\prime}+2 V\left(1+\frac{f^{\prime 2}}{2 f}\right)-\left(S_{\text {matt }}-E_{\text {matt }}\right)\left(1+\frac{f^{\prime 2}}{f}\right)\right]\right\},
\end{aligned}
$$

where $S_{\text {matt }}^{a b}:=\gamma^{a}{ }_{c} \gamma^{b}{ }_{d} T_{\text {matt }}^{c d}$ and $S_{\text {matt }}$ is its trace.

Finally, Eq. (2.8) can be written as the following first order evolution equation [2]

$$
\mathcal{L}_{n} \Pi-\Pi K-Q^{c} D_{c}[\ln \alpha]-D_{c} Q^{c}=-\frac{f V^{\prime}-2 f^{\prime} V-\frac{1}{2} f^{\prime}\left(1+3 f^{\prime \prime}\right)\left(Q^{2}-\Pi^{2}\right)+\frac{1}{2} f^{\prime} T_{\text {matt }}}{f\left(1+\frac{3 f^{\prime 2}}{2 f}\right)},
$$

where $T_{\text {matt }}=S_{\text {matt }}-E_{\text {matt }}$. Here one must remember that indices of 3-tensors (including 3-vectors) are raised and lowered with the 3-metric $\gamma$, and that the contravariant time components of such quantities are identically null. This is why one is usually only interested in the spatial components of 3-tensors.

A useful evolution equation for the trace of the extrinsic curvature takes the form

$$
\begin{aligned}
\partial_{t} K & -\beta^{l} \partial_{l} K+{ }^{3} \Delta \alpha-\alpha K_{i j} K^{i j}-\frac{\alpha f^{\prime}}{f}\left(D_{l} Q^{l}+\Pi K\right)-\frac{\alpha}{f\left(1+\frac{3 f^{\prime 2}}{2 f}\right)}\left\{\Pi^{2}\left(1+\frac{3 f^{\prime 2}}{4 f}+\frac{3 f^{\prime \prime}}{2}\right)+Q^{2}\left[\frac{3 f^{\prime 2}}{4 f}\left(1+2 f^{\prime \prime}\right)-\frac{f^{\prime \prime}}{2}\right]\right\} \\
& =\frac{\alpha}{2 f\left(1+\frac{3 f^{\prime 2}}{2 f}\right)}\left\{S_{\text {matt }}+E_{\text {matt }}\left(1+\frac{3 f^{\prime 2}}{f}\right)-2 V\left(1-\frac{3 f^{\prime 2}}{2 f}\right)-3 f^{\prime} V^{\prime}\right\}
\end{aligned}
$$

where ${ }^{3} \Delta:=D^{l} D_{l}$ is the Laplacian compatible with the 3metric, and $D_{c} Q^{c}=\partial_{c} Q^{c}+Q^{c} \partial_{c}(\ln \sqrt{\gamma}) \quad$ (here $\gamma=$ $\left.\operatorname{det} \gamma_{i j}\right)$.

The system of Eqs. (2.12), (2.13), and (2.14) can now be rewritten as

$$
\begin{gathered}
\partial_{t} \phi=\alpha \Pi+\beta^{a} Q_{a}, \\
\partial_{t} Q_{i}-\beta^{l} \partial_{l} Q_{i}-Q_{l} \partial_{i} \beta^{l}=D_{i}(\alpha \Pi) . \\
\partial_{t} \gamma_{i j}=-2\left(\alpha K_{i j}-D_{(i} \beta_{j}\right),
\end{gathered}
$$

This system of evolution equations is to be completed with appropriate evolution equation for the gauge variables (lapse and shift). This issue is considered next.

In order to obtain a closed evolution system, one has to impose gauge conditions for the time variable $t$ and for the spatial coordinates $x^{i}$. In this work we shall consider a modified Bona-Massó time slicing defined in the following way (cf. Ref. [2]):

$$
\square t=\left(\frac{1}{f_{\mathrm{BM}}}-1\right) n^{b} n^{c} \nabla_{b} \nabla_{c} t+\frac{\Theta}{f_{\mathrm{BM}}} \frac{f^{\prime}}{f} \Pi n^{a} \nabla_{a} t,
$$

with $f_{\mathrm{BM}}=f_{\mathrm{BM}}(\alpha)>0$ a positive but otherwise arbitrary function of the lapse, and $\Theta=\Theta\left(f_{\mathrm{BM}}\right)$ is in principle an arbitrary function of $f_{\mathrm{BM}}$ which can a posteriori be fixed so as to ensure a well behaved hyperbolic system (see Sec. III below). It is important not to confuse the gauge function $f_{\mathrm{BM}}(\alpha)$ with the NMC $f(\phi)$.

Concerning the spatial coordinates, we shall consider the shift vector as an a priori known function of the coordinates. However, in the future it would be interesting to investigate some "live" shift conditions and their effects in phenomena involving STT.

Now, the slicing condition (2.23) for local coordinates $x^{a}=\left(t, x^{i}\right)$ adapted to the $3+1$ foliation of the spacetime reduces to

$$
\frac{d \alpha}{d t}=-\alpha^{2} f_{\mathrm{BM}}\left(K-\frac{\Theta}{f_{\mathrm{BM}}} \frac{f^{\prime}}{f} \Pi\right),
$$

where $d / d t:=\partial_{t}-\mathcal{L}_{\boldsymbol{\beta}}$. The specific choices $\Theta=f_{\mathrm{BM}} \equiv$ 1 correspond to a modified harmonic slicing condition (termed "pseudoharmonic" in [2]), which was specially useful for the second order hyperbolicity analysis performed in [2]. On the other hand, with $\Theta \equiv 0$ one recovers the usual BM slicing condition. Just like in standard GR, we shall see that one can take in general any positive $f_{\mathrm{BM}}$ (not necessarily $f_{\mathrm{BM}} \equiv 1$ ) to obtain a well behaved strongly hyperbolic system. Moreover, the choice $\Theta=1$ or $\Theta=f_{\text {BM }}$ provides simple sufficient conditions for regular eigenfields in the STT, as we will show below. An inadequate choice for $\Theta$ leads to eigenfields that can be ill-defined when $f_{\mathrm{BM}}=1$ locally or globally (cf. Eqs. (3.24) and (3.49) below). A bad choice can be 
precisely $\Theta=0$, so the usual BM slicing condition seems not to be well adapted for constructing strongly hyperbolic formulations of STT's in the Jordan frame.

\section{HYPERBOLIC SYSTEMS}

In the previous section, we showed a constrained system of first order in time partial differential equations (PDE) corresponding to the STT. This system is well formulated in the sense that one can construct an unambiguous numerical algorithm that evolves initial data satisfying the constraint equations. In the case of a globally null scalar field, the above system of equations reduces to the usual ADMY system of equations of GR. Even if such a system is well formulated in the above sense, it is well-known that the evolution is not stable in many cases.

It took several years to realize that such instabilities were not associated with the numerical algorithms but rather with the mathematical structure of the ADMY system itself. During the mid-1990's, many researchers started to suspect that the numerical instabilities that seemed to plague several test bed codes based in the usual $3+1$ formalism were caused by the lack of (strong) hyperbolicity of the system. Therefore, many investigations were devoted to develop new strongly hyperbolic formulations of the $3+1$ evolution equations of GR (see [9] and references therein). However, at that time, it was still not totally clear that such new hyperbolic formulations presented more computational advantages than the usual ADMY system. The breakthrough towards a real improvement of the numerical stability was the use of a novel formulation of GR which used a conformal formalism, today known as the BSSN formulation $[9,10]$. In [9] it was recognized that the new conformal system presented some hyperbolic features not present in the original ADMY system, and moreover it was stressed that the use of suitably defined connection coefficients and the subsequent addition of the momentum constraints (in order to eliminate some divergence operators) proved to be essential for numerical stability. Nonetheless, it was after a more systematic analysis of the BSSN system that a much better understanding of its hyperbolicity properties was obtained [21]. In connection with the hyperbolicity properties of the $3+1$ system, one needs to mention the gauge choice (i.e., the prescription of the evolution of the gauge variables). In the formal developments and proofs of theorems about the wellposedness of the Cauchy problem of GR, it was the use of the harmonic gauge that allowed one to write the Einstein field equations as a second order diagonal hyperbolic system of quasilinear PDE's. Nevertheless, in numerical implementations, the use of the (pure) harmonic gauge has proven not to be very successful, specially in the strong gravity regime where one requires to avoid (or freeze) the formation of singularities in the numerical grid. A big step towards solving this problem was the implementation of new gauge conditions (e.g., the so- called Bona-Massó time slicing) in hyperbolic formulations [7]. Currently, many hyperbolic formulations, together with Bona-Massó like slicing conditions, have been proposed in the literature.

In this and the following sections, we will propose two systems for the STT based on the $3+1$ system of Sec. II but written in fully first order form along the lines of the BSSN and the BMSS formulations, and show that they are indeed strongly hyperbolic. Our aim for constructing two strongly hyperbolic formulations of the STT is to understand the way in which the modified Bona-Massó condition (2.24) enters into both formulations, and how robust is this new class of slicing conditions. In fact, we will see that both formulations are compatible with the choices $\Theta=1$, $f_{\mathrm{BM}}$ in that the eigenfields turn out to be well behaved without imposing any further stringent conditions on the gauge function $f_{\mathrm{BM}}$ (apart from it being positive definite).

Let us first outline the general approach we intend to follow for both formulations. From the numerical point of view, it seems to be more useful to recast a second order system of PDE of hyperbolic type into a full first order form as follows

$$
\partial_{t} \vec{u}+\mathbb{M}^{i} \partial_{i} \vec{u}=\vec{S}(\vec{u}),
$$

where $\vec{u}$ represents collectively the fundamental variables (like the $\gamma_{i j}$ 's, $K_{i j}$ 's, etc.). $\mathbb{M}^{i}$ are called the characteristic matrices of the system, and $\vec{S}(\vec{u})$ represents source terms which include only the fundamental variables but not their derivatives. This system of PDE is said to be quasilinear since it is linear in the derivatives but in general is nonlinear in the $\vec{u}$.

A quasilinear system of PDE of the form (3.1) possesses a well-posed Cauchy problem (in the Hadamard sense) only in very special circumstances (see Refs. [22,23] for a review), which depend on the structure of the characteristic matrices $\mathbb{M}^{i}$. In general, when one deals with such a system one can choose a specific spatial direction of propagation of fields along which one analyzes the hyperbolic character of the system. For instance, if the direction of propagation is specified by a unit covector $v_{i}$, then the hyperbolic nature of the system will be determined by the matrix $\mathbb{C}:=v_{i} \mathbb{M}^{i}$, known as the principal symbol. Namely, the system will be weakly hyperbolic or strongly hyperbolic, if the following properties are satisfied respectively: 1) $\mathbb{C}$ has real eigenvalues but an incomplete set of eigenvectors; 2) $\mathbb{C}$ has real eigenvalues and a complete set of eigenvectors (for any $v_{i}$ ). In addition, the system is said to be symmetric or symmetrizable hyperbolic, respectively, if: 3) $\mathbb{M}^{i}$ are symmetric, 4) $\mathbb{M}^{i}$ can be symmetrized. Only strongly, symmetric, and symmetrizable hyperbolic systems of PDE admit a well-posed Cauchy problem.

The systematic approach to analyze the hyperbolicity of a system of evolution equations along these lines consists then in first recasting the system in the form (3.1), and then solving the eigenvalue problem of the corresponding prin- 
cipal symbol. If $\mathbb{C}$ satisfies the conditions of 2) above, then one can ensure well-posedness. In general, one finds that for the systems of evolution equations found in the $3+1$ formulation of GR, it is necessary to add multiples of the constraint Eqs. (2.15) and (2.16) to some of the evolution equations in order to make the system strongly hyperbolic [24]. This procedure does not affect the physical solutions (i.e., those that satisfy the constraint equations), but it does affect the structure of the $\mathbb{C}$ matrix.

Now, suppose that the following system is strongly hyperbolic in a given direction $v_{i}$ [i.e., in what follows we will assume that $\mathbb{C}$ satisfies the condition 2) above]. From Eq. (3.1) one finds that

$$
\partial_{t} \vec{u}+\mathbb{C} \nabla_{v} \vec{u} \simeq 0,
$$

where $\nabla_{v}$ stands for the directional derivative along $v_{i}$, and $\simeq$ indicates that we shall focus only in the principal part of (3.1). If we define now $\mathbb{R}$ as the matrix of eigenvectors of $\mathbb{C}$, then $\mathbb{L}=\mathbb{R}^{-1} \mathbb{C} \mathbb{R}$ is the diagonal eigenvalue matrix. Notice that $\mathbb{R}$ can clearly be inverted since we have assumed that $\mathbb{C}$ has a complete set of eigenvectors. Multiplication of (3.2) on the left by $\mathbb{R}^{-1}$ leads to the equivalent system

$$
\partial_{t} \vec{w}+\mathbb{L} \nabla_{s} \vec{w} \simeq 0,
$$

where we have introduced the functions $\vec{w}:=\mathbb{R}^{-1} \vec{u}$ which are called the eigenfields or eigenfunctions. Clearly, the new system (3.3) is now decoupled and each eigenfield $w_{i}$ propagates with its own characteristic speed given by its corresponding eigenvalue $\lambda_{i}$.

All this construction depends heavily on the existence of a complete set of eigenvectors (in addition to the existence of real eigenvalues). The system (3.2) is therefore equivalent to (3.3) when the strongly hyperbolicity conditions are met. Furthermore, conditions on the smoothness of the eigenvectors (or eigenfields) and several other conditions concerning the gauge speeds (e.g., real-valued speeds, singularity avoidance) impose in turn conditions on the class of gauges adopted in the selection of the time slicing (see below) and the spatial coordinates (although here we shall consider the shift condition as a prescribed one).

Now, it turns out that very often (depending on the complexity of the system) one can construct the equivalent system (3.3) not by using direct methods to diagonalize the matrices $\mathbb{M}^{i}$, but instead by a "judicious guessing" approach which consists in constructing the eigenfields and corresponding eigenvalues by inspection (see Appendix B for a simple example). This is for instance the approach followed in [7]. In Sec. III A and III B below we use this inspection approach to construct the eigenfields and their corresponding speeds of propagation.

\section{A. Bona-Massó Formulation of STT}

In order to construct a full first order system based on the system of equations of Sec. II, we first need to define new first order variables in the following way

$$
a_{i}=\partial_{i} \ln \alpha, \quad d_{k i j}=\frac{1}{2} \partial_{k} \gamma_{i j} .
$$

Moreover, we will also introduce the auxiliary variable

$$
V_{i}=d_{i m}{ }^{m}-d_{m i}^{m}
$$

where $d_{i j}{ }^{l}:=\gamma^{l m} d_{i j m}$ and $d^{l}{ }_{i j}:=\gamma^{l m} d_{m i j}$. Using this definition, the principal part of ${ }^{3} R_{i j}$ reads

$$
{ }^{3} R_{i j} \simeq-\partial_{m} d^{m}{ }_{i j}-\partial_{(i}\left(2 V_{j)}-d_{j) m}{ }^{m}\right) .
$$

With such definitions the evolution Eqs. (2.17) will be of first order in space for $a_{i}$ and $d_{k i j}$, instead of second order for the lapse and the 3-metric.

After some algebra, one obtains the following set of 34 first order evolution equations, written here only up to principal part (the full form of the equations can be seen in Appendix A)

$$
\begin{gathered}
\partial_{0} Q_{i} \simeq \alpha \partial_{l}\left(\delta_{i}^{l} \Pi\right), \\
\partial_{0} \Pi \simeq \alpha \partial_{l} Q^{l}, \\
\partial_{0} a_{i} \simeq-\alpha \partial_{l}\left[\delta_{i}^{l}\left(f_{\mathrm{BM}} K-\Theta \frac{f^{\prime}}{f} \Pi\right)\right], \\
\partial_{0} d_{i j k} \simeq-\alpha \partial_{i} K_{j k}, \\
\partial_{0} K_{i j} \simeq-\alpha \partial_{l} \Lambda_{i j}^{l}, \\
\partial_{0} V^{i} \simeq \alpha \partial_{l}\left[(1+\varsigma)\left(K^{i l}-\gamma^{i l} K\right)+\mathrm{s} \gamma^{i l} \frac{f^{\prime}}{f} \Pi\right],
\end{gathered}
$$

where $\partial_{0}:=\partial_{t}-\beta^{l} \partial_{l}, V^{i}:=\gamma^{i l} V_{l}$, and

$$
\Lambda_{i j}^{l}:=d_{i j}^{l}+\delta_{(i}^{l}\left(a_{j)}+\frac{f^{\prime}}{f} Q_{j)}+2 V_{j)}-d_{j) m}{ }^{m}\right) \text {. }
$$

Though the $\Lambda_{i j}^{l}$ are not independent variables, it is nevertheless useful to have their evolution equation which take the form

$$
\begin{aligned}
\partial_{0} \Lambda_{i j}^{l} \simeq & -\alpha \gamma^{l m} \partial_{m} K_{i j}+\alpha \delta_{(i}^{l} \partial_{m}\left[2(1+\varsigma)\left(K^{m}{ }_{j}-\delta^{m}{ }_{j} K\right)\right. \\
& \left.+\delta^{m}{ }_{j)}\left(1-f_{\mathrm{BM}}\right) K+\frac{f^{\prime}}{f} \Pi \delta^{m}{ }_{j)}(1+\Theta+2 \varsigma)\right] .
\end{aligned}
$$

Here we must stress the fact that in Eq. (3.12) we have added a multiple $\alpha$ s of the momentum constraint (2.16). Moreover, the evolution equations for the lapse and the 3metric were not included since they are "trivial" in the sense that the lapse and the 3-metric do not evolve up to principal part and only they propagate along the "normal lines." As pointed out in [25], the above evolution system, Eqs. (3.7), (3.8), (3.9), (3.10), (3.11), and (3.12), can be 
seen as a reduced system evolving in a inhomogeneous "background."

Now, in order to obtain the eigenfields propagating in one specific direction, we will consider the $x$ direction, i.e., $v_{i}=(1,0,0)$, and ignore the derivatives in the other directions (cf. [6]). This is equivalent to analyzing only the characteristic matrix $\mathbb{M}^{x}$. Thus, for $q \neq x$, it is clear from the evolution Eqs. (3.7), (3.8), (3.9), (3.10), (3.11), and (3.12) that there are 18 eigenfunctions which propagate with speed $-\beta^{x}$, namely $w_{1,2}=Q_{q}$ (corresponding to the two eigenfields propagating in the two directions orthogonal to $x$ ), $w_{3,4}=a_{q}, w_{5-16}=d_{q j k}$ (12 eigenfields, six for each $q)$, and $w_{17,18}=V_{q}+(1+\varsigma) d_{l q}^{l}$.

Furthermore, by using the evolution equations we appreciate that the following combinations

$$
\begin{aligned}
& w_{19}=a_{x}-f_{\mathrm{BM}} d_{x m}{ }^{m}-\Theta \frac{f^{\prime}}{f} Q_{x}, \\
& w_{20}=V^{x}-\varsigma \frac{f^{\prime}}{f} Q^{x}-(1+\varsigma) \hat{\Lambda}^{x},
\end{aligned}
$$

provide two more eigenfunctions propagating along the normal lines with $-\beta^{x}$ speed, where

$$
\hat{\Lambda}^{x}:=\Lambda^{x}-\frac{\Lambda^{x x x}}{\gamma^{x x}}=\left(\gamma^{x x} \gamma^{p q}-\gamma^{x p} \gamma^{x q}\right) \frac{\Lambda_{p q}^{x}}{\gamma^{x x}}
$$

is the projection of $\Lambda_{p q}^{x}$ on the surface $x=$ const (for $q \neq$ $x$ and $p \neq x$ ) [6]. Here we used $\Lambda^{x}:=\gamma^{l m} \Lambda^{x}{ }_{l m}$ and $\Lambda^{x x x}:=\gamma^{x l} \gamma^{x m} \Lambda_{l m}^{x}$. Such a projection is in fact achieved with $\left(P^{x}\right)_{i j}:=\gamma_{i j}-\left(s^{x}\right)_{i}\left(s^{x}\right)_{j}$, where $\vec{s}^{x}$ is the normal to the surface with components given by $\left(s^{x}\right)_{i}=\delta_{i}^{x} / \sqrt{\gamma^{x x}}$ and $\left(s^{x}\right)^{i}=\gamma^{x i} / \sqrt{\gamma^{x x}}$. So $\hat{\Lambda}^{x}=\left(P^{x}\right)^{i j} \Lambda^{x}{ }_{i j}$. In fact by projecting Eq. (3.14) on the surface $x=$ const, and focusing in propagation only in the $x$ direction, one obtains $\partial_{0} \hat{\Lambda}^{x} \simeq$ $-\alpha \gamma^{x x} \partial_{x} \hat{K}$, where $\hat{K}:=\left(P^{x}\right)^{i j} K_{i j}$ whose expression is given below in Eq. (3.25).

Raising the index in Eq. (3.7), and combining the evolution equation for $Q^{x}$ with Eq. (3.8), we obtain the following two eigenfunctions

$$
w_{21,22}=Q^{x} \pm \sqrt{\gamma^{x x}} \Pi,
$$

which propagate along the light cones with eigenvalues

$$
\lambda_{ \pm}=-\beta^{x} \mp \alpha \sqrt{\gamma^{x x}} .
$$

Next, combining the equations for $K_{q p}$ and $\Lambda_{q p}^{x}$, we form the following six more eigenfunctions:

$$
w_{23-28}=\Lambda_{p q}^{x} \pm \sqrt{\gamma^{x x}} K_{p q},
$$

which propagate also along the light cones with eigenvalues

$$
\sigma_{ \pm}=-\beta^{x} \pm \alpha \sqrt{\gamma^{x x}} .
$$

Combining the expressions for $K_{q}^{x}$ and $\Lambda_{q}^{x x}\left(\Lambda_{q}^{x x}:=\right.$ $\gamma^{x l} \Lambda_{l q}^{x}$, one can see that

$$
w_{29-32}=\Lambda_{q}^{x x} \pm \sqrt{-\varsigma \gamma^{x x}} K^{x}{ }_{q}
$$

are four more eigenfunctions with eigenvalues

$$
\omega_{ \pm}=-\beta^{x} \pm \alpha \sqrt{-\varsigma \gamma^{x x}} .
$$

Finally the last two eigenfunctions turn out to be

$$
\begin{aligned}
w_{33,34}= & \Lambda^{x}+\frac{2(1+\varsigma)}{f_{\mathrm{BM}}-1} \hat{\Lambda}^{x} \pm \sqrt{f_{\mathrm{BM}} \gamma^{x x}}\left[K+\frac{2(1+\varsigma)}{f_{\mathrm{BM}}-1} \hat{K}\right] \\
& +\left(\frac{2 \varsigma+1+\Theta}{f_{\mathrm{BM}}-1}\right) \frac{f^{\prime}}{f}\left(Q^{x} \mp \sqrt{f_{\mathrm{BM}} \gamma^{x x}} \Pi\right)
\end{aligned}
$$

where

$$
\hat{K}:=K-\frac{K^{x x}}{\gamma^{x x}}=\left(\gamma^{x x} \gamma^{p q}-\gamma^{x p} \gamma^{x q}\right) \frac{K_{p q}}{\gamma^{x x}}
$$

is defined in a similar fashion as $\hat{\Lambda}^{x}$ (cf. Eq. (3.17)).

The eigenfields $w_{33,34}$ propagate with the "gauge speeds" given by the following eigenvalues

$$
\delta_{ \pm}=-\beta^{x} \pm \alpha \sqrt{f_{\mathrm{BM}} \gamma^{x x}} .
$$

We have then 34 linearly independent eigenfunctions $w_{1-34}$ which are equivalent to the 34 linearly independent variables $\left(Q_{i}, \Pi, a_{i}, d_{i j k}, K_{i j}, V^{i}\right)$, so the system is strongly hyperbolic.

From the 34 eigenfunctions obtained above, we appreciate that only $w_{33,34}$ might develop gauge divergences, while the remaining eigenfields are smooth (as long as the original variables themselves are smooth) regardless of the time slicing. Note, for instance, how in Eq. (3.24) the choice $f_{\mathrm{BM}} \equiv 1$ can cause those eigenfunctions to blow up unless we choose the value $\varsigma=-1$ (see the coefficients of $\hat{\Lambda}^{x}$ and $\hat{K}$ in $\left.w_{33,34}\right)$. Moreover, even with $\varsigma=-1$, another blowup of the same sort might appear in the coefficient with $\frac{f^{\prime}}{f}$ of $w_{33,34}$. However, that problem can in turn be avoided by a suitable choice for $\Theta$. The simplest choices are $\Theta=1$ and $\Theta=f_{\mathrm{BM}}$. For instance, the eigenfunctions $w_{33,34}$ with $\mathrm{s}=-1$ and $\Theta=1$ simply become

$$
w_{33,34}=\Lambda^{x} \pm \sqrt{f_{\mathrm{BM}} \gamma^{x x}} K .
$$

Moreover, with $\varsigma=-1$ the eigenfield (3.16) becomes

$$
w_{20}=V^{x}+\frac{f^{\prime}}{f} Q^{x} .
$$

The value $\varsigma=-1$ also turns out to be compatible with the condition for real-valued eigenfunctions $w_{29-32}$ [cf. Eq. (3.22)]. Now, when one chooses $f_{\mathrm{BM}} \equiv 1$, the two optimal values for $\Theta$ reduce both to $\Theta=1$, and the slicing condition (2.24) is compatible with the modified harmonic time slicing which together with the modified harmonic spatial coordinates (both written as $\square x^{a}=$ $-\frac{1}{f} \nabla^{a} f$ ) were the ones required in Ref. [2] to reduce the 
field equations of STT (without matter) into a quasilinear diagonal second order hyperbolic system. Furthermore, note that $w_{33,34}$ degenerate $\left(w_{33}=w_{34}\right)$ if $f_{\mathrm{BM}}=0$, and the eigenfields are not complete in that case. We then appreciate the importance of the condition $f_{\mathrm{BM}}>0$. In particular, in the BMSS formulation of standard GR, the condition $f_{\mathrm{BM}} \geq 1$ seemed to be the most adequate for a convenient mode propagation and singularity avoidance behavior.

Finally, let us remark that in pure GR the eigenfield (3.28) is given by $w_{20}=V^{x}$ which propagates along the normal lines. We just saw that $w_{17,18}=V_{q}$ (with $\left.\varsigma=-1\right)$ also propagate along the normal lines. Therefore in pure GR all three $V_{i}$ 's propagate along the normal lines. In pure GR there are also only 30 eigenfields $\left(w_{1,2}\right.$ and $w_{21,22}$ are absent), and in this limit all the eigenfields coincide or are equivalent to the ones obtained in Ref. [6], which are precisely the eigenfields found in the Bona-Massó formulation. As emphasized in Ref. [6], in GR taking $s=0$ as opposed to $s=-1$ (which is the value proposed in the Bona-Massó formulation) is equivalent to the full first order ADMY system. That is, $s=0$ amounts to not adding the momentum constraints to the evolution equations. The ADMY system is then weakly hyperbolic. This feature is manifest from Eq. (3.22) in that one has two less independent eigenfields with $s=0$, so that one cannot reconstruct the original 30 variables $\left(a_{i}, d_{i j k}, K_{i j}, V^{i}\right)$ of GR. We then conclude that for STT $\varsigma=0$ also leads to a weakly hyperbolic system since then one has only 32 eigenfunctions from which it is impossible to reconstruct the 34 field variables listed above.

\section{B. BSSN Formulation of STT}

The BSSN formulation of GR $[9,10]$ is perhaps the most popular and useful formulation for numerical approaches in use today. This formulation was found to be much more stable in numerical simulations than the standard ADMY approach. A further analysis of the BSSN formulation revealed that this is in fact a strongly hyperbolic system [21]. Although it is clear that the strong hyperbolicity property is a key ingredient that causes BSSN to perform better than the ADMY formulation (which is only weakly hyperbolic), this feature seems to be not the only issue in the assessment. In fact there are other strongly hyperbolic formulations that perform poorly in comparison with BSSN. Apparently it is the conformal approach that provides another important ingredient to the numerical stability. Nevertheless, it is still not very well understood why the conformal approach makes such an improvement relative to other strongly hyperbolic formulations. More recently, the BSSN formulation has also shown great stability in binary black hole simulations when the moving puncture method is implemented [26,27]. Notably, this formulation is able to evolve the initial data of two inspiraling black holes until the merger stage and beyond without the code crashing. This was a long standing problem in numerical relativity. Moreover, this formulation has also been very successful when matter is included (see for instance [2830]).

In view of these advantages, we propose here an analog of the BSSN formulation but extended to the STT. Moreover, we will also deal with the proposed modified Bona-Massó slicing condition in this formulation and study the roll played by the function $\Theta$ in the construction of well behaved eigenfields. Just like in Sec. III A, we shall define new variables so as to obtain a full first order system (both in time and space), but following now the conformal approach of BSSN.

We consider again the first of Eqs. (3.4), and introduce the following variables

$$
\begin{gathered}
\tilde{\gamma}_{i j}=e^{-4 \psi} \gamma_{i j}, \\
\tilde{A}_{i j}=e^{-\zeta \psi} A_{i j}, \\
\tilde{d}_{i j k}=\frac{1}{2} \partial_{i} \tilde{\gamma}_{j k}, \\
\Psi_{i}=\partial_{i} \psi, \\
{ }^{3} \tilde{\Gamma}^{i}=\tilde{\gamma}^{j k 3} \tilde{\Gamma}^{i}{ }_{j k}=-\partial_{j} \tilde{\gamma}^{i j},
\end{gathered}
$$

where $\psi$ is chosen such that the determinant $\tilde{\gamma}$ of the conformal metric is equal to one [31], ${ }^{3} \tilde{\Gamma}_{j k}^{i}$ are the 3 Christoffel symbols associated with the conformal metric, $\zeta$ is a constant, and $A_{i j}$ is the trace-free part of $K_{i j}$ given by

$$
A_{i j}=K_{i j}-\frac{1}{3} \gamma_{i j} K
$$

From the evolution system of Sec. II, one obtains the following conformal evolution system up to principal part (see Appendix A for the full system of equations):

$$
\begin{gathered}
\partial_{0} Q_{i} \simeq \alpha \partial_{l}\left(\delta_{i}^{l} \Pi\right), \\
\partial_{0} \Pi \simeq \alpha \partial_{l} Q^{l}, \\
\partial_{0} a_{i} \simeq-\alpha \partial_{l}\left[\delta_{i}^{l}\left(f_{\mathrm{BM}} K-\Theta \frac{f^{\prime}}{f} \Pi\right),\right. \\
\partial_{0} \tilde{d}_{i j k} \simeq-\alpha e^{(\zeta-4) \psi} \partial_{i} \tilde{A}_{j k}, \\
\partial_{0} \Psi_{i} \simeq-\frac{1}{6} \alpha \partial_{i} K, \\
\partial_{0} K \simeq-\alpha e^{-4 \psi} \tilde{\gamma}^{j l} \partial_{l}\left(a_{j}-\frac{f^{\prime}}{f} Q_{j}\right), \\
\partial_{0} \tilde{A}_{i j} \simeq-\alpha e^{-\zeta \psi} \partial_{l} \tilde{\Lambda}_{i j}^{l},
\end{gathered}
$$




$$
\partial_{0}{ }^{3} \tilde{\Gamma}^{i} \simeq \alpha \partial_{l}\left[(\xi-2) e^{(\zeta-4) \psi} \tilde{A}^{i l}+\tilde{\gamma}^{i l} \xi\left(-\frac{2}{3} K+\frac{f^{\prime}}{f} \Pi\right)\right] .
$$

Again, we stress that in Eq. (3.42) above we have added a multiple $\alpha \xi$ of the momentum constraints (2.16) to the evolution equation for the variable ${ }^{3} \tilde{\Gamma}^{i}$. Notice also $\tilde{A}^{i j}=$ $\tilde{\gamma}^{i l} \tilde{\gamma}^{j m} \tilde{A}_{l m}=e^{(8-\zeta) \psi} A^{i j}$, and we have also defined

$$
\tilde{\Lambda}_{i j}^{l}:=\left[\tilde{d}_{i j}^{l}+\delta_{(i}^{l}\left(a_{j)}+\frac{f^{\prime}}{f} Q_{j)}-{ }^{3} \tilde{\Gamma}_{j)}+2 \Psi_{j)}\right)\right]^{\mathrm{TF}},
$$

where TF means that the quantity between the brackets is trace-free (i.e., $\tilde{\gamma}^{i j} \tilde{\Lambda}_{i j}^{l} \equiv 0$ ). Here $\tilde{d}_{i j}^{l}:=\tilde{\gamma}^{l m} \tilde{d}_{m i j}$ and ${ }^{3} \tilde{\Gamma}_{j}:=\tilde{\gamma}_{j l}{ }^{3} \tilde{\Gamma}^{l}$. For completeness we provide the evolution equation for $\tilde{\Lambda}_{i j}^{l}$

$$
\begin{aligned}
\partial_{0} \tilde{\Lambda}_{i j}^{l} \simeq & -\alpha\left\{e^{(\zeta-4) \psi} \partial_{m}\left[\tilde{\gamma}^{l m} \tilde{A}_{i j}+(\xi-2) \delta_{(i}^{l} \tilde{A}_{j)}^{m}\right]\right. \\
& +\left(f_{\mathrm{BM}}+\frac{1-2 \xi}{3}\right) \delta_{(i}^{l} \partial_{j)} K \\
& \left.-\frac{f^{\prime}}{f}(\Theta+1-\xi) \delta_{(i}^{l} \partial_{j)} \Pi\right\}^{\mathrm{TF}} .
\end{aligned}
$$

In the above system of evolution equations we consider only the reduced system since the lapse and the 3-metric propagate along the normal lines.

Like in the BM hyperbolic formulation of Sec. III A, there are again 34 variables, namely, 15 linearly independent $\tilde{d}_{i j m}$, five linearly independent $\tilde{A}_{i j}$ [32], three ${ }^{3} \tilde{\Gamma}^{i}$, one $K$, three $\Psi_{i}$ (for the derivatives of the conformal factor), three $a_{i}$, and finally, four variables $\Pi$ and $Q_{i}$ related to the derivatives of the scalar field.

In order to find the eigenfields associated with these 34 variables, we consider again a particular direction of propagation, say $i=x$. For $q \neq x$, it is clear from the above evolution equations that there are again 18 eigenfunctions which propagate with speed $-\beta^{x}$, namely $w_{1,2}=$ $Q_{q}, w_{3,4}=a_{q}, w_{5,6}=\Psi_{q}$, and $w_{7-16}=\tilde{d}_{q j k}$.

Furthermore, we appreciate that the following combinations

$$
\begin{gathered}
w_{17}=a_{x}-6 f_{\mathrm{BM}} \Psi_{x}-\Theta \frac{f^{\prime}}{f} Q_{x}, \\
w_{18-20}={ }^{3} \tilde{\Gamma}^{i}+(\xi-2) \tilde{d}_{m}{ }^{m i}-\xi \tilde{\gamma}^{i k}\left(4 \Psi_{k}+\frac{f^{\prime}}{f} Q_{k}\right),
\end{gathered}
$$

provide another four eigenfunctions with speed $-\beta^{x}$, where $\tilde{d}_{m}{ }^{m i}:=\tilde{\gamma}^{i j} \tilde{\gamma}^{m l} \tilde{d}_{m l j}$. Thus, we have obtained 20 eigenfunctions.

Moreover, just like in Sec. III A, we obtain another two eigenfunctions from the following combinations

$$
w_{21,22}=Q^{x} \pm \sqrt{\gamma^{x x}} \Pi,
$$

with eigenvalues

$$
\lambda_{ \pm}=-\beta^{x} \mp \alpha \sqrt{\gamma^{x x}} .
$$

Next, combining the evolution equations for $K, Q^{x}, a^{x}$, and $\Pi$, we form the following two eigenfunctions:

$$
\begin{aligned}
w_{23,24}= & a^{x}-\left(\frac{\Theta-f_{\mathrm{BM}}}{1-f_{\mathrm{BM}}}\right) \frac{f^{\prime}}{f} Q^{x} \\
& \pm e^{-2 \psi} \sqrt{f_{\mathrm{BM}}} \tilde{\gamma}^{x x}\left[K+\left(\frac{\Theta-1}{1-f_{\mathrm{BM}}}\right) \frac{f^{\prime}}{f} \Pi\right]
\end{aligned}
$$

with eigenvalues

$$
\sigma_{ \pm}=-\beta^{x} \pm \alpha e^{-2 \psi} \sqrt{f_{\mathrm{BM}} \tilde{\gamma}^{x x}} .
$$

Combining the evolution equations for $\tilde{\Lambda}_{q}^{x x}\left(\tilde{\Lambda}_{j}^{l i}:=\right.$ $\tilde{\gamma}^{i k} \tilde{\Lambda}_{k j}^{l}$ ) and $\tilde{A}_{q}^{x}$, one appreciates that

$$
w_{25-28}=\tilde{\Lambda}_{q}^{x x} \pm e^{(\zeta-2) \psi} \sqrt{\frac{\xi}{2}} \tilde{\gamma}^{x x} \tilde{A}_{q}^{x}
$$

are four more eigenfunctions with eigenvalues

$$
\omega_{ \pm}=-\beta^{x} \pm \alpha e^{-2 \psi} \sqrt{\frac{\xi}{2} \tilde{\gamma}^{x x}} .
$$

Furthermore, combining $\tilde{\Lambda}^{x x x}\left(\tilde{\Lambda}^{i j k}:=\tilde{\gamma}^{l k} \tilde{\Lambda}^{i j}{ }_{l}\right), \tilde{\Lambda}^{x}{ }_{q p}, \tilde{A}^{x x}$, and $\tilde{A}_{q p}$, we obtain the following eigenfunctions:

$$
\begin{aligned}
w_{29-32}= & \tilde{\Lambda}_{p q}^{x}+\frac{\tilde{\gamma}_{p q}}{2 \tilde{\gamma}^{x x}} \tilde{\Lambda}^{x x x} \\
& \pm e^{(\zeta-2) \psi} \sqrt{\tilde{\gamma}^{x x}}\left(\tilde{A}_{p q}+\frac{\tilde{\gamma}_{p q}}{2 \tilde{\gamma}^{x x}} \tilde{A}^{x x}\right)
\end{aligned}
$$

with eigenvalues

$$
\delta_{ \pm}=-\beta^{x} \pm \alpha e^{-2 \psi} \sqrt{\tilde{\gamma}^{x x}} .
$$

As remarked in [6], $w_{29-32}$ are in fact only four independent eigenfunctions since these are symmetric and surface traceless.

Finally the last 2 eigenfunctions are obtained by combining the evolution equations for $\tilde{\Lambda}^{x x x}, a^{x}, Q^{x}, \tilde{A}^{x x}, K$, and $\Pi$

$$
\begin{aligned}
w_{33,34}= & \tilde{\Lambda}^{x x x}-\frac{1}{3} \tilde{\gamma}^{x x}\left(2 \tilde{a}^{x}+\frac{f^{\prime}}{f} \tilde{Q}^{x}\right) \\
& \pm e^{2 \psi} \sqrt{\tilde{\gamma}^{x x} \frac{2 \xi-1}{3}\left[e^{(\zeta-4) \psi} \tilde{A}^{x x}\right.} \\
& \left.+\tilde{\gamma}^{x x}\left(-\frac{2}{3} K+\frac{f^{\prime}}{f} \Pi\right)\right],
\end{aligned}
$$

where $\tilde{a}^{x}:=\tilde{\gamma}^{x l} a_{l}$ and $\tilde{Q}^{x}:=\tilde{\gamma}^{x l} Q_{l}$. These propagate with the following speeds 


$$
\eta_{ \pm}=-\beta^{x} \pm \alpha e^{-2 \psi} \sqrt{\tilde{\gamma}^{x x} \frac{2 \xi-1}{3}}
$$

Thus, we have found the complete set of eigenfunctions and eigenvalues. The 34 linearly independent eigenfields $w_{1-34}$ are equivalent to the 34 linearly independent variables $\left(Q_{i}, \Pi, a_{i}, \tilde{d}_{i j k}, \Psi_{i}, K, \tilde{A}_{i j},{ }^{3} \tilde{\Gamma}^{i}\right)$.

Now, like in the Bona-Massó system of Sec. III A, there are some eigenfunctions (namely $w_{23,24}$ ) that might blow up when $f_{\mathrm{BM}}=1$. In order to avoid this problem, while maintaining the possible choice $f_{\mathrm{BM}} \equiv 1$, one has two simple possibilities: $\Theta=1, f_{\mathrm{BM}}$. These are the same conditions found in Sec. III A for well behaved eigenfields. For instance, with $\Theta=1$ the eigenfunctions $w_{23,24}$ reduce to

$$
w_{23,24}=a^{x}-\frac{f^{\prime}}{f} Q^{x} \pm e^{-2 \psi} \sqrt{f_{\mathrm{BM}} \tilde{\gamma}^{x x}} K,
$$

with the same eigenvalues, and the blowup disappears even if $f_{\mathrm{BM}}=1$. The same happens with the choice $\Theta=f_{\mathrm{BM}}$, and thus we conclude again that in either case $(\Theta=1$, $f_{\mathrm{BM}}$ ), the choice $f_{\mathrm{BM}} \equiv 1$ requires $\Theta=1$ which leads, as emphasized before, to the modified harmonic slicing condition $\square t=-\frac{1}{f} \partial^{t} f$ [2] [cf. Equation (2.23) with $\Theta=$ $\left.f_{\mathrm{BM}} \equiv 1\right]$.

The remaining eigenfunctions are smooth for any $f_{\mathrm{BM}}>$ 0 and the eigenvalues are real valued. Note, on the other hand, that if $f_{\mathrm{BM}}=0$ the eigenfunctions $w_{23,24}$ degenerate and then the system is no longer complete.

None of the eigenvalues depend on $\zeta$, and the values $\zeta=$ 2,4 seem the optimal since in that case many exponential factors cancel out $(\zeta=4$ is the one used in the original BSSN formulation of GR, while $\zeta=-2$ has been used for constructing initial data [19]). On the other hand, from Eqs. (3.55) and (3.56) we appreciate that the eigenfunctions and the eigenvalues are real and non-degenerate only for $\xi>1 / 2$, and therefore the system is strongly hyperbolic if this condition holds. A particular case corresponds to the value $\xi=2$ which seems to be the most convenient since in that case the eigenfunctions $w_{18-20}$ [see Eq. (3.46)], $w_{25-28}$ [see Eq. (3.51)], and $w_{33,34}$ [see Eq. (3.55)] are simpler. In fact this is the value used in the original BSSN formulation of GR. We remark that taking $\xi=0$ corresponds to the case where the momentum constraint is not added to the evolution equation for ${ }^{3} \tilde{\Gamma}^{i}$ [cf. Eq. (3.42)], and that choice makes the system not even weakly hyperbolic since the eigenvalues (3.56) become imaginary. This is a feature that was already present in pure GR [6]. Actually, when the scalar field $\phi$ is absent, the hyperbolicity analysis presented in this section is similar (for $\zeta=4$ and $\xi=2$ ) to the corresponding analysis of the standard BSSN formulation [6]. Finally, it is important to mention that in order to avoid any possible divergence in the eigenfields or in the source terms (the terms not contributing to the principal part of the evolution equations) of both formulations, one needs to consider only STT with $f(\phi)>0$ (i.e., a positive definite NMC function). This in turn precludes the possibility of having an infinite or negative effective gravitational constant $G_{\text {eff }}$ [cf. Eqs. (2.7)].

\section{DISCUSSION}

In this paper, we have constructed two novel first order strongly hyperbolic formulations of the STT in the Jordan frame along the lines of the BM and BSSN approaches. Such constructions show that both formulations have a well-posed Cauchy problem. This analysis fills the gap of a previous study on the Cauchy problem of STT [2] and confirms that the Jordan frame is mathematically adequate for treating the initial value problem.

One of the most interesting features of the formulations presented here is that a modified Bona-Massó slicing condition is required for the two new systems to be strongly hyperbolic while allowing several slicings $\left(f_{\mathrm{BM}}>0\right)$ which are natural generalizations of the slicings used in pure GR. In particular $\Theta=1, f_{\mathrm{BM}}$ in Eq. (2.24) are two simple choices that lead to well behaved eigenfields.

In the absence of a scalar field, the equations of Sec. III A and III B reduce (for $\varsigma=-1, \zeta=4$, and $\xi=$ 2 ) to the known BM and BSSN formulations of GR (when the NMC function is trivial, i.e., $F(\phi) \equiv 1$, the "gravitational" and the scalar-field sectors decouple completely up to principal part).

What remains to be investigated is the usefulness and robustness of these formulations in actual numerical experiments, as well as the inclusion of a "live shift." Actually, we plan to analyze the dynamical transition to the phenomenon of spontaneous scalarization in boson stars arising in STT [33] and the subsequent gravitational collapse to a black hole with gravitational wave emission of scalar type, using one or both of the hyperbolic formulations presented here. Both phenomena (spontaneous scalarization and scalar gravitational waves) are even present in spherical symmetry due to the NMC, therefore by assuming such a symmetry one can simplify the equations without eliminating the interesting physical features.

An important consequence of the analysis presented here is that a slightly more general STT which includes a function $\omega(\phi)$ in the kinetic term of the scalar-field sector of the action (2.1) (i.e., the kinetic term has the form $\left.\omega(\phi)(\nabla \phi)^{2} / 2\right)$ posses a well-posed Cauchy problem as well (except for some choices of $\omega(\phi)$; see Ref. [34]). The fact is that all the terms with $\omega(\phi)$ do not contribute to the principal part of the equations associated with the metric sector [i.e., the equivalent of Eq. (2.2)], while the scalarfield sector [the equivalent of Eq. (2.8)] preserves the quasilinear diagonal hyperbolic form (see Ref. [34] for the detailed equations). Thus, up to the principal part such STT are identical to the ones analyzed here. The relevance of this generalization is that such STT can be mapped to the so-called modified theories of gravity which are given by a Lagrangian density $f(R)(R$ being the Ricci 
scalar) [34]. Some specific choices of $f(R)$ lead to gravity theories which have been recently analyzed in several contexts. Notably, in the cosmological setting such theories have been proposed as an alternative to dark energy, since they can produce an accelerating expansion of the Universe without any exotic form of matter [35,36]. However, it must be emphasized that some of these theories might violate the Solar System tests [37,38], and some modifications are required to circumvent such drawbacks (see Ref. [39] for a review). The point we want to underline here is that, from the mathematical point of view, the viability of such theories relies heavily on the wellposedness of the Cauchy problem.

\section{ACKNOWLEDGMENTS}

This work was supported in part by CONACyT Grants No. SEP-2004-C01-47209-F and No. 149945, and by DGAPA-UNAM Grants No. IN112401 and No. IN119005. D. M. acknowledges support from CONACyT.

\section{APPENDIX A: FULL SYSTEM OF EQUATIONS}

The full system of evolution equations for the generalized Bona-Massó formulation of STT is the following:

$$
\begin{gathered}
\frac{d}{d t} Q_{i}=D_{i}(\alpha \Pi), \\
\frac{d}{d t} \Pi=\alpha \Pi K+\alpha Q^{c} D_{c} \ln (\alpha)+\alpha D_{c} Q^{c} \\
-\frac{\alpha}{f\left(1+\frac{3 f^{\prime 2}}{2 f}\right)}\left[f V^{\prime}-2 f^{\prime} V-\frac{1}{2} f^{\prime}\left(1+3 f^{\prime \prime}\right)\right. \\
\left.\times\left(Q^{2}-\Pi^{2}\right)+\frac{1}{2} f^{\prime} T_{\mathrm{matt}}\right], \\
\frac{d}{d t} \alpha=-\alpha^{2}\left(f_{\mathrm{BM}} K-\Theta \frac{f^{\prime}}{f} \Pi\right), \\
\frac{d}{d t} a_{i}=-\partial_{i}\left[\alpha\left(f_{B M} K-\Theta \frac{f^{\prime}}{f} \Pi\right)\right] \\
\frac{d}{d t} \gamma_{i j}=-2 \alpha K_{i j}, \\
\frac{d}{d t} K_{i j}=-D_{i} D_{j} \alpha+\alpha^{3} R_{i j}+\alpha K K_{i j}-2 \alpha K_{i l} K_{j}^{l} \\
+4 \pi G_{0} \alpha\left[\gamma_{i j}(S-E)-2 S_{i j}\right], \\
+2 d_{i l(j)} \partial_{k)} \beta^{l},
\end{gathered}
$$

$$
\begin{aligned}
\partial_{0} V^{i}= & -V^{l} \partial_{l} \beta^{i}+(1+\varsigma) D_{l}\left[\alpha\left(K^{i l}-\gamma^{i l} K\right)\right] \\
& +\frac{1}{2}\left(\partial^{i} \partial_{m} \beta^{m}-\partial^{m} \partial_{m} \beta^{i}\right)-\varsigma \alpha\left[a_{j}\left(K^{i j}-\gamma^{i j} K\right)\right. \\
& \left.+8 \pi G_{0} J^{i}\right]+\alpha K^{j k}\left(\delta^{i}{ }_{j}{ }^{3} \Gamma_{k l}^{l}-{ }^{3} \Gamma^{i}{ }_{j k}\right),
\end{aligned}
$$

where we remind the notations $d / d t:=\partial_{t}-\mathcal{L}_{\boldsymbol{\beta}}$ and $\partial_{0}:=\partial_{t}-\beta^{l} \partial_{l}$.

In fact, this last equation can also be written as

$$
\begin{aligned}
\partial_{0} V_{i}= & V_{l} \partial_{i} \beta^{l}+\alpha(1+\varsigma) \partial_{l}\left(K_{i}^{l}-\delta_{i}^{l} K\right) \\
& +\alpha a_{j}\left(K^{j}{ }_{i}-\delta^{j}{ }_{i} K\right)+\frac{1}{2}\left(\partial_{m i}^{2} \beta^{m}-\gamma_{i j} \gamma^{m l} \partial_{m l}^{2} \beta^{j}\right) \\
& -\alpha K^{j k}\left\{\gamma_{i j}\left[2 V_{k}-(2+\varsigma)^{3} \Gamma_{k l}^{l}\right]+{ }^{3} \Gamma_{i j k}\right. \\
& \left.+(1+\varsigma)^{3} \Gamma_{k i j}\right\}-\varsigma \alpha 8 \pi G_{0} J_{i},
\end{aligned}
$$

where we note that ${ }^{3} \Gamma^{i}{ }_{j k}=\gamma^{i l}\left(2 d_{(j k) l}-d_{l j k}\right)$ and ${ }^{3} \Gamma_{k l}^{l}=$ $\gamma^{j l} d_{k j l}=\partial_{k}(\ln \sqrt{\gamma})$. So for the optimal choice $s=-1$, Eq. (A9) reduces to the usual equation of the BMSS formulation (cf. Eq. (8) of Ref. [40]).

We also write here the following terms which contain the contributions of the scalar and matter fields [2]:

$$
\begin{gathered}
4 \pi G_{0} \alpha\left[(S-E) \gamma_{i j}-2 S_{i j}\right] \\
=-\frac{\alpha}{f}\left[Q_{i} Q_{j}\left(1+f^{\prime \prime}\right)+f^{\prime}\left(D_{i} Q_{j}+\Pi K_{i j}\right)+S_{i j}^{\text {matt }}\right] \\
+\frac{\gamma_{i j} \alpha}{2 f\left(1+\frac{3 f^{\prime 2}}{2 f}\right)}\left[\left(Q^{2}-\Pi^{2}\right)\left(\frac{f^{\prime 2}}{2 f}-f^{\prime \prime}\right)-f^{\prime} V^{\prime}\right. \\
\left.-2 V\left(1+\frac{f^{\prime 2}}{2 f}\right)+\left(S_{\text {matt }}-E_{\text {matt }}\right)\left(1+\frac{f^{\prime 2}}{f}\right)\right], \quad(\mathrm{A} 10) \\
\quad \times\left\{\Pi^{2}\left(1+\frac{3 f^{\prime 2}}{4 f}+\frac{3 f^{\prime \prime}}{2}\right)\right. \\
+Q_{0} \alpha(S+E)= \\
\left.\left.\alpha \frac{f^{\prime}}{f}\left(D_{m} Q^{m}+\Pi K\right)+\frac{\alpha f^{\prime 2}}{4 f}\left(1+2 f^{\prime \prime}\right)-\frac{f^{\prime \prime}}{2}\right]\right\} \\
+\frac{\alpha}{2 f\left(1+\frac{3 f^{\prime 2}}{2 f}\right)}\left\{S_{\text {matt }}+E_{\text {matt }}\left(1+\frac{3 f^{\prime 2}}{f}\right)\right. \\
\left.-2 V\left(1-\frac{3 f^{\prime 2}}{2 f}\right)-3 f^{\prime} V^{\prime}\right\}, \\
8 \pi G_{0} J_{i}=\frac{1}{f}\left[-f^{\prime}\left(K_{m i} Q^{m}+D_{i} \Pi\right)-\Pi Q_{i}\left(1+f^{\prime \prime}\right)\right. \\
\left.+J_{i}^{\text {matt }}\right]
\end{gathered}
$$

The 3-Ricci tensor has the explicit form 


$$
\begin{aligned}
{ }^{3} R_{i j}= & \partial_{l}{ }^{3} \Gamma^{l}{ }_{i j}-\partial_{i}{ }^{3} \Gamma^{l}{ }_{j l}+{ }^{3} \Gamma^{m}{ }_{i j}{ }^{3} \Gamma^{l}{ }_{m l}-{ }^{3} \Gamma^{l}{ }_{i m}{ }^{3} \Gamma^{m}{ }_{l j} \\
= & -\partial_{m} d^{m}{ }_{i j}-\partial_{(i}\left(2 V_{j)}-d_{j) m}{ }^{m}\right)-4 d^{m}{ }_{m k} d_{(i j)}{ }^{k} \\
& +4 d^{l}{ }_{m(i} d_{j) l}{ }^{m}-d_{i l}{ }^{m} d_{j m}{ }^{l}+2 d^{l}{ }_{m i}\left(d_{l j}{ }^{m}-d^{m}{ }_{l j}\right) \\
& +d_{l m}{ }^{m}\left(2 d_{(i j)}{ }^{l}-d^{l}{ }_{(i j)}\right) .
\end{aligned}
$$

This expression can be easily rewritten in the form used by the BMSS formulation (cf. Eq. (10) of Ref. [40]).

On the other hand, the full system of evolution equations for the generalized BSSN formulation of STT is the following:

$$
\begin{aligned}
& \frac{d}{d t} Q_{i}=D_{i}(\alpha \Pi), \\
& \frac{d}{d t} \Pi=\alpha \Pi K+\alpha Q^{c} D_{c} \ln (\alpha)+\alpha D_{c} Q^{c}-\frac{\alpha}{f\left(1+\frac{3 f^{\prime 2}}{2 f}\right)} \\
& \times\left[f V^{\prime}-2 f^{\prime} V-\frac{1}{2} f^{\prime}\left(1+3 f^{\prime \prime}\right)\left(Q^{2}-\Pi^{2}\right)\right. \\
& \left.+\frac{1}{2} f^{\prime} T_{\text {matt }}\right] \text {, } \\
& \frac{d}{d t} \alpha=-\alpha^{2}\left(f_{\mathrm{BM}} K-\Theta \frac{f^{\prime}}{f} \Pi\right), \\
& \partial_{0} \psi=-\frac{1}{6}\left(\alpha K-\partial_{l} \beta^{l}\right), \\
& \frac{d}{d t} a_{i}=-\partial_{i}\left[\alpha\left(f_{\mathrm{BM}} K-\Theta \frac{f^{\prime}}{f} \Pi\right)\right], \\
& \frac{d}{d t} \tilde{\gamma}_{i j}=-2 \alpha e^{(\zeta-4) \psi} \tilde{A}_{i j} \\
& \partial_{0} \tilde{d}_{i j k}=-\partial_{i}\left(\alpha e^{(\zeta-4) \psi} \tilde{A}_{j k}\right)+\tilde{d}_{l j k} \partial_{i} \beta^{l}+2 \tilde{d}_{i l(j} \partial_{k)} \beta^{l} \\
& -\frac{2}{3} \tilde{d}_{i j k} \partial_{l} \beta^{l}-\frac{1}{3} \tilde{\gamma}_{j k} \partial_{i l}^{2} \beta^{l}+\tilde{\gamma}_{l(j} \partial_{k) i}^{2} \beta^{l}, \\
& \partial_{0} \Psi_{i}=-\frac{1}{6} \partial_{i}\left(\alpha K-\partial_{l} \beta^{l}\right)+\Psi_{l} \partial_{i} \beta^{l}, \\
& \frac{d}{d t} K=-D_{i} D^{i} \alpha+\alpha\left(e^{2(\zeta-4) \psi} \tilde{A}_{i j} \tilde{A}^{i j}+\frac{1}{3} K^{2}\right) \\
& +4 \pi G_{0} \alpha(E+S), \\
& \frac{d}{d t} \tilde{A}_{i j}=e^{-\zeta \psi}\left[-D_{i} D_{j} \alpha+\alpha\left({ }^{3} R_{i j}-8 \pi G_{0} S_{i j}\right)\right]^{\mathrm{TF}} \\
& +\alpha\left[\left(\frac{\zeta+2}{6}\right) K \tilde{A}_{i j}-2 e^{(\zeta-4) \psi} \tilde{A}_{i k} \tilde{A}_{j}^{k}\right],
\end{aligned}
$$

where $S_{i j}^{\mathrm{TF}}$ can be extracted from Eq. (A10). In order to have a full first order formulation it is understood that the second order spatial derivatives of the lapse and the physical 3-metric appearing in the above equations have to be written, respectively, in terms of first derivatives of $a_{i}, \tilde{d}_{i j k}$, and $\Psi_{i}$.

The evolution equation for the ${ }^{3} \tilde{\Gamma}^{i}$ is

$$
\begin{aligned}
\partial_{0}{ }^{3} \tilde{\Gamma}^{i}= & \alpha(\xi-2) e^{(\zeta-4) \psi} \partial_{l} \tilde{A}^{i l}-\alpha \xi\left(\frac{2}{3} \tilde{\gamma}^{i l} \partial_{l} K+8 \pi G_{0} \tilde{J}^{i}\right) \\
& +2 \alpha e^{(\zeta-4) \psi} \tilde{A}^{l m}\left\{\left[\frac{\xi}{2}(\zeta+2)+4-\zeta\right] \Psi_{l} \delta^{i}{ }_{m}\right. \\
& \left.-\delta^{i}{ }_{m} a_{l}+\frac{\xi}{2}{ }^{3} \tilde{\Gamma}^{i}{ }_{l m}\right\}+\tilde{\gamma}^{j k} \partial_{j k}^{2} \beta^{i}+\frac{1}{3} \tilde{\gamma}^{i j} \partial_{k j}^{2} \beta^{k} \\
& -{ }^{3} \tilde{\Gamma}^{j} \partial_{j} \beta^{i}+\frac{2}{3}{ }^{3} \tilde{\Gamma}^{i} \partial_{j} \beta^{j},
\end{aligned}
$$

where $\tilde{J}^{i}:=\tilde{\gamma}^{i l} J_{l}$ whose explicit expression can be obtained from Eq. (A12).

We stress that the above equations reduce to the corresponding equations of the original BSSN formulation for $\zeta=4$ and $\xi=2$ [41].

We must note that for tensor densities $\mathcal{D}$ of weight $w$ (here we omit the rank) the Lie derivative has an extra weight term

$$
\mathcal{L}_{\boldsymbol{\beta}} \mathcal{D}=\left[\mathcal{L}_{\boldsymbol{\beta}} \mathcal{D}\right]_{w=0}+w \mathcal{D} \partial_{m} \beta^{m},
$$

where the weight $w$ is related to the power of the determinant of the physical metric as $\gamma^{w / 2}$, and the first term on the right-hand side of Eq. (A25) is to be understood as the ordinary Lie derivative (as though $\mathcal{D}$ were a true tensor). For instance, in the case of $\tilde{\gamma}_{i j}$ and $\tilde{A}_{i j}$, we see from Eqs. (3.29) and (3.30) that these are $(0,2)$ tensor densities of weight $-2 / 3$ and $-\zeta / 6$, respectively, so

$$
\begin{aligned}
\mathcal{L}_{\boldsymbol{\beta}} \mathcal{D}_{i j}= & \beta^{m} \partial_{m} \mathcal{D}_{i j}+\mathcal{D}_{i m} \partial_{j} \beta^{m}+\mathcal{D}_{m j} \partial_{i} \beta^{m} \\
& +w \mathcal{D}_{i j} \partial_{m} \beta^{m},
\end{aligned}
$$

where $\mathcal{D}_{i j}$ stands for $\tilde{\gamma}_{i j}$ and $\tilde{A}_{i j}$ with $w=-2 / 3$ and $w=$ $-\zeta / 6$, respectively.

For completeness, we provide the expression of the 3Ricci tensor ${ }^{3} R_{i j}$ in terms of the conformal metric (3.29)

$$
{ }^{3} R_{i j}={ }^{3} \tilde{R}_{i j}+{ }^{3} R_{i j}^{\psi}
$$

where

$$
\begin{aligned}
{ }^{3} \tilde{R}_{i j}= & -\frac{1}{2} \tilde{\gamma}^{l m} \partial_{l m}^{2} \tilde{\gamma}_{i j}+\tilde{\gamma}_{k(i} \partial_{j}{ }^{3} \tilde{\Gamma}^{k}+{ }^{3} \tilde{\Gamma}^{k 3} \tilde{\Gamma}_{(i j) k} \\
& +\tilde{\gamma}^{l m}\left(2^{3} \tilde{\Gamma}^{k}{ }_{l(i}{ }^{3} \tilde{\Gamma}_{j) k m}+{ }^{3} \tilde{\Gamma}^{k}{ }_{i m}{ }^{3} \tilde{\Gamma}_{k l j}\right), \\
{ }^{3} R_{i j}^{\psi}:= & -2 \tilde{D}_{i} \tilde{D}_{j} \psi-2 \tilde{\gamma}_{i j} \tilde{D}^{k} \tilde{D}_{k} \psi+4\left(\tilde{D}_{i} \psi\right)\left(\tilde{D}_{j} \psi\right) \\
- & 4 \tilde{\gamma}_{i j}\left(\tilde{D}^{k} \psi\right)\left(\tilde{D}_{k} \psi\right),
\end{aligned}
$$

where $\tilde{D}$ stands for the covariant derivative associated with the conformal metric $\tilde{\gamma}^{i j}$, and ${ }^{3} \tilde{\Gamma}_{k i j}:=\tilde{\gamma}_{k l}{ }^{3} \tilde{\Gamma}^{l}{ }_{i j}$. In fact by putting tildes in Eq. (A13) and noticing that $\tilde{d}_{k m}{ }^{m}=$ ${ }^{3} \tilde{\Gamma}_{k l}^{l}=\partial_{k}(\ln \sqrt{\tilde{\gamma}}) \equiv 0 \quad$ (since $\tilde{\gamma}:=1$ ), we see that Eq. (A28) can be written as Eq. (A13) with the terms 
involving $\tilde{d}_{l m}{ }^{m}$ dropped. Moreover, the constraint Eqs. (2.15) and (2.16) can be easily rewritten in terms of the quantities defined in Eqs. (3.29), (3.30), (3.31), (3.32), (3.33), and (3.34).

\section{APPENDIX B: SIMPLE EXAMPLE OF AN HYPERBOLIC SYSTEM}

Let us consider the system

$$
\begin{gathered}
\partial_{t} u_{1}+a \partial_{x} u_{1}+b \partial_{x} u_{2}=S_{u_{1}}\left(u_{1}, u_{2}\right), \\
\partial_{t} u_{2}+c \partial_{x} u_{1}+d \partial_{x} u_{2}=S_{u_{2}}\left(u_{1}, u_{2}\right),
\end{gathered}
$$

where the coefficients $a-d$ are in general functions of $(t, x)$, and the $S_{u_{1}, u_{2}}\left(u_{1}, u_{2}\right)$ are source functions. Using the judicious guessing approach we shall construct a new system of the form (3.3) which is manifestly hyperbolic, and then we shall confront this method with the more systematic method which uses linear algebra.

First, we ask for a linear combination $w:=u_{1}+\sigma u_{2}$ to be the eigenfunction. This means that we write an evolution equation for $w$, where $\sigma(a, b, c, d)$ is a function of the coefficients $a-d$, such that the right-hand side takes the form $-\lambda_{\sigma} \partial_{x} w$, where $\lambda_{\sigma}$ will be the eigenvalue. In this way we have

$$
\begin{aligned}
\partial_{t}\left(u_{1}+\sigma u_{2}\right) & \simeq-\partial_{x}\left[(a+\sigma c) u_{1}+(b+\sigma d) u_{2}\right] \\
& \simeq-(a+\sigma c) \partial_{x}\left(u_{1}+\frac{b+\sigma d}{a+\sigma c} u_{2}\right) .
\end{aligned}
$$

Note that with the symbol $\simeq$ we have discarded from the analysis all the terms which do not contain derivatives of $u_{1}$ and $u_{2}$ (including the sources). In this way, we can assume as that the coefficients $a-d$ are "constants." By comparing the coefficients of $u_{2}$ on both sides of Eq. (B3), we see that we need to take $\sigma=(b+\sigma d) /(a+\sigma c)$, which implies $\sigma_{ \pm}=\frac{d-a \pm \sqrt{(a-d)^{2}+4 b c}}{2 c}$. Moreover, the eigenfunctions will be smooth provided $c(t, x)$ does not vanish in some spacetime point.

Therefore we conclude that the two eigenfunctions are $w_{ \pm}=u_{1}+\sigma_{ \pm} u_{2}$ with the corresponding eigenvalues $\lambda_{ \pm}=a+\sigma_{ \pm} c=\frac{a+d \pm \sqrt{(a-d)^{2}+4 b c}}{2}$. The eigenvalues are real and the eigenfunctions are nondegenerate if $(a-$ $d)^{2}+4 b c>0$ and $c \neq 0$. If this condition holds and the eigenfields are smooth then the system is strongly hyperbolic [42].

Now, let us find the eigenfunctions of the same system using the standard method of linear algebra. We rewrite the system in a more convenient matrix form as

$$
\partial_{t}\left(\begin{array}{l}
u_{1} \\
u_{2}
\end{array}\right)+\left(\begin{array}{ll}
a & b \\
c & d
\end{array}\right) \partial_{x}\left(\begin{array}{l}
u_{1} \\
u_{2}
\end{array}\right) \simeq 0 .
$$

The eigenvalues $\lambda$ of the characteristic matrix turn out to be $\lambda_{ \pm}=\frac{a+d \pm \sqrt{(a-d)^{2}+4 b c}}{2}$, which are identical to the ones found before. On the other hand, the eigenvectors are

$$
v_{+}=\left(\begin{array}{c}
-\sigma_{-} \\
1
\end{array}\right), \quad v_{-}=\left(\begin{array}{c}
\sigma_{+} \\
-1
\end{array}\right)
$$

Thus, the matrix of eigenvectors reads

$$
\mathbb{R}=\left(\begin{array}{cc}
-\sigma_{-} & \sigma_{+} \\
1 & -1
\end{array}\right)
$$

Then we can write the eigenfunctions as

$$
\left(\begin{array}{l}
f_{1} \\
f_{2}
\end{array}\right)=\mathbb{R}^{-1}\left(\begin{array}{l}
u_{1} \\
u_{2}
\end{array}\right)=\frac{1}{\sigma_{+}-\sigma_{-}}\left(\begin{array}{l}
u_{1}+\sigma_{+} u_{2} \\
u_{1}+\sigma_{-} u_{2}
\end{array}\right)
$$

which are the same found before modulo the factor $\frac{1}{\sigma_{+}-\sigma_{-}}=c / \sqrt{(a-d)^{2}+4 b c}$. The fact that we do not obtain exactly the same eigenfunctions is a consequence of the fact that the eigenvectors are unique only up to a rescaling. However, both pairs of eigenfunctions $w_{ \pm}$and $f_{1,2}$ are equivalent.

Finally, notice that if $a=d$, then $\lambda_{ \pm}=a \pm \sqrt{b c}$, and $w_{ \pm}=u_{1} \pm \sqrt{b / c} u_{2}$. Thus $a$ plays the same roll as $-\beta^{i}$ in the evolution equations of Sec. II. For instance, if the coefficients $b, c$ are null, the Eqs. (B1) and (B2) decouple and then $u_{1}$ and $u_{2}$ are themselves eigenfunctions, indicating that they propagate along the "normal lines" (with speed $a$ ). This is precisely what happens with eigenfunctions $w_{1-18}$ of Sec. III A. On the other hand, when $b$ and $c$ are non-null, these coefficients are related to the propagation of the eigenfields some of which propagate along the "light cones." For instance, if $b$ is identified with $\alpha \gamma^{x x}$ (or $-\varsigma \alpha \gamma^{x x}$, or even $\alpha f_{\mathrm{BM}} \gamma^{x x}$ ) and $c$ with $\alpha$, in many of the evolution equations of Sec. III A (assuming propagation only in the $x$ direction), then the $\lambda_{ \pm}$are in turn to be identified with the speeds of propagation $-\beta^{x} \pm \alpha \sqrt{\gamma^{x x}}$ or $-\beta^{x} \pm \alpha \sqrt{-\varsigma} \gamma^{x x} \quad$ or even $-\beta^{x} \pm \alpha \sqrt{f_{\mathrm{BM}} \gamma^{x x}}$ (cf. Eqs. (3.21), (3.23), and (3.26), respectively) of several eigenfields (e.g., $\quad w_{ \pm}=u_{1} \pm \sqrt{\gamma^{x x}} u_{2}, \quad$ or $\quad w_{ \pm}=$ $u_{1} \pm \sqrt{-\varsigma} \gamma^{x x} u_{2}$, or even $w_{ \pm}=u_{1} \pm \sqrt{f_{\mathrm{BM}} \gamma^{x x}} u_{2}$, where $u_{1}$ and $u_{2}$ represent field variables like those entering in the expressions for $w_{23-28}, w_{29-32}$, and $w_{33,34}$, respectively).
[1] V. Faraoni, Cosmology in Scalar-Tensor Gravity (Kluwer Academic Publishers, Dordrecht, The Netherlands, 2004).
[2] M. Salgado, Classical Quantum Gravity 23, 4719 (2006).

[3] R.M. Wald, General Relativity (The University of 
Chicago Press, Chicago, 1984).

[4] R. Arnowitt, S. Deser, and C. W. Misner, in Gravitation: An Introduction to Current Research, edited by L. Witten (John Wiley, New York, 1962), pp. 227-265.

[5] J. York, in Sources of Gravitational Radiation, edited by L. Smarr (Cambridge University Press, Cambridge, England, 1979).

[6] M. Alcubierre, "Introduction to $3+1$ Numerical Relativity," (Oxford University Press, Oxford, to be published).

[7] C. Bona, J. Massó, E. Seidel, and J. Stela, Phys. Rev. Lett. 75, 600 (1995).

[8] C. Bona and J. Massó, Phys. Rev. Lett. 68, 1097 (1992).

[9] T.W. Baumgarte and S.L. Shapiro, Phys. Rev. D 59, 024007 (1998).

[10] M. Shibata and T. Nakamura, Phys. Rev. D 52, 5428 (1995).

[11] C.M. Will, Theory and Experiment in Gravitational Physics (Cambridge University Press, Cambridge, England, 1993).

[12] M. Salgado, D. Sudarsky, and H. Quevedo, Phys. Rev. D 53, 6771 (1996).

[13] M. Salgado, D. Sudarsky, and H. Quevedo, Phys. Lett. B 408, 69 (1997).

[14] M. Salgado, D. Sudarsky, and H. Quevedo, Astrophys. J. 488, 14 (1997).

[15] H. Quevedo, M. Salgado, and D. Sudarsky, Gen. Relativ. Gravit. 31, 767 (1999).

[16] M. Salgado, D. Sudarsky, and U. Nucamendi, Phys. Rev. D 58, 124003 (1998).

[17] T. Damour and G. Esposito-Farèse, Phys. Rev. D 54, 1474 (1996).

[18] Here and in what follows, Latin indices from the first letters of the alphabet $a, b, c, \ldots$ are four-dimensional and run $0-3$, while Latin indices starting from $i(i, j, k, \ldots)$ are three-dimensional and run 1-3.

[19] E. Gourgoulhon, arXiv:gr-qc/0703035.

[20] A slightly different notation to that of Ref. [2] has been used here in order to match with the one used in many references on numerical relativity. In order to return to the notation of [2], one needs to perform the transformation $\alpha \rightarrow N, \beta^{i} \rightarrow-N^{i}, \gamma_{i j} \rightarrow h_{i j}$ for the lapse, shift and the 3-metric, respectively.

[21] O. Sarbach, G. Calabrese, J. Pullin, and M. Tiglio, Phys. Rev. D 66, 064002 (2002).

[22] O. Reula, arXiv:gr-qc/0403007.

[23] O. A. Reula, Living Rev. Relativity 1, 3 (1998), http:// www.livingreviews.org/lrr-1998-3.

[24] L. E. Kidder, M. A. Scheel, and S. A. Teukolsky, Phys. Rev. D 64, 064017 (2001).

[25] C. Bona, J. Massó, E. Seidel, and J. Stela, Phys. Rev. D 56, 3405 (1997).

[26] M. Campanelli, C. O. Lousto, P. Marronetti, and Y. Zlochower, Phys. Rev. Lett. 96, 111101 (2006).

[27] J. G. Baker, J. Centrella, D.-I. Choi, M. Koppitz, and J. van
Meter, Phys. Rev. Lett. 96, 111102 (2006).

[28] Y. T. Liu, S. L. Shapiro, and B. C. Stephens, Phys. Rev. D 76, 084017 (2007).

[29] L. Baiotti, I. Hawke, and L. Rezzolla, Classical Quantum Gravity 24, S187 (2007).

[30] M. Shibata, Y. T. Liu, S. L. Shapiro, and B. C. Stephens, Phys. Rev. D 74, 104026 (2006).

[31] In fact, as suggested in Ref. [19], when one works with coordinates other that Cartesian-type, it turns out to be better to define $\psi$ so that $\operatorname{det} \tilde{\gamma}_{i j}=\operatorname{det} \tilde{f}_{i j}$, where $f_{i j}$ represents a background Riemannian metric. For instance if $\Sigma_{t}$ is asymptotically flat one can take $f_{i j}$ to be the flat metric in some coordinates adapted to the symmetry of the problem. In spherical symmetry, say, $f_{i j}=$ $\operatorname{diag}\left(1, r^{2}, r^{2} \sin ^{2} \theta\right)$ and $\operatorname{det} \tilde{\gamma}_{i j}=r^{4} \sin ^{2} \theta$. This approach has the advantage that $\psi$ becomes a true scalar while all the tensorial quantities defined using $\tilde{\gamma}_{i j}$ and $\psi$ become true tensors instead of tensor densities.

[32] We remind the reader that $\tilde{A}_{i j}$ and $\tilde{d}_{i j m}$ have only 5 and 15 linearly independent components, respectively, since both are traceless. In fact, from Eq. (3.31) we conclude that $\tilde{\gamma}^{j m} \tilde{d}_{i j m}=\frac{1}{2} \partial_{i}\left(\operatorname{det} \tilde{\gamma}_{i j}\right) \equiv 0$, since one chooses $\tilde{\gamma}_{i j}$ so that $\operatorname{det} \tilde{\gamma}_{i j} \equiv 1$.

[33] A. W. Whinnett, Phys. Rev. D 61, 124014 (2000).

[34] N. Lanahan-Tremblay and V. Faraoni, Classical Quantum Gravity 24, 5667 (2007).

[35] S. M. Carroll, V. Duvvuri, M. Trodden, and M. S. Turner, Phys. Rev. D 70, 043528 (2004).

[36] S. Capozziello, S. Carloni, and A. Troisi, Res. Dev. Astron. Astrophys. 1, 625 (2003).

[37] A. L. Erickcek, T. L. Smith, and M. Kamionkowski, Phys. Rev. D 74, 121501 (2006).

[38] T. Chiba, T. L. Smith, and A. L. Erickcek, Phys. Rev. D 75, 124014 (2007).

[39] S. Nojiri and S. D. Odintsov, Int. J. Geom. Methods Mod. Phys. 4, 115 (2007).

[40] A. Arbona, C. Bona, J. Massó, and J. Stela, Phys. Rev. D 60, 104014 (1999).

[41] For comparison purposes with the original BSSN equations, a typo in sign has to be taken into account in the second line of Eq. (24) of [9].

[42] If the function $c(t, x)$ is globally null, the equation for $u_{2}$ decouples and $u_{2}$ is itself an eigenfunction propagating with speed $d$; the other eigenvalue and eigenfunction can be obtained by proceeding in the same way, taking $c=0$ in Eq. (B3). This results in $w_{2}=u_{1}+b u_{2} /(a-d)$ (with $a \neq d$ ) and $\lambda_{2}=a$. However, if $c=0, a=d$, and $b \neq 0$ then the system degenerates and becomes weakly hyperbolic. In such a case the characteristic matrix is a Jordan block [cf. Eq. (B4)], which implies that it cannot be diagonalized. A similar situation happens if $b=0, a=$ $d$, and $c \neq 0$. On the other hand, if $c=0=b$ then both Eqs. (B1) and (B2) decouple and therefore $u_{1}$ and $u_{2}$ are themselves eigenfunctions (or any linear combination of them is an eigenfunction as well if in addition $a=d$ ). 